\section{Management of Optic Disc Melanocytoma Associated with Choroidal Neovascularisation and Macular Detachment}

\author{
Vaishnavi Batmanabane*, Manoj Soman and Unnikrishnan \\ Nair
}

Department of Retina and Vitreous, Chaithanya Eye Hospital and Research Institute, Kesavadasapuram, Trivandrum, Kerala, India

\begin{abstract}
Purpose: An optic disc melanocytoma is often considered a benign lesion, requiring only routine follow-up to assess possible progression to a malignant condition or development of a Choroidal Neovascular Membrane (CNVM) which can cause a drop in vision and eventually loss of sight if untreated. With the advent of imaging technology, the follow-up and monitoring of disease progression and therapeutic interventions have been made easier and more comprehensive. We investigated a patient with this lesion using Spectral Domain-optical Coherence Tomography (SD-OCT), autofluorescence imaging, fundus fluorescein angiography, and documented her response to treatment with intravitreal ranibizumab for secondary CNVM.

Keywords: Optic disc melanocytoma, Ranibizumab, Spectral Domain-optical Coherence Tomography
\end{abstract}

\section{Introduction}

An optic disc melanocytoma is characteristically a benign lesion [1], with a potential for malignant transformation [2,3]. These dark brown to black colored tumors are usually located on the optic nerve head, and many a time, the asymptomatic nature [4] of this condition prompts discovery only on routine ophthalmic examination. Several complications such as retinal vein obstruction [5,6] optic nerve infiltration [7], tumor enlargement $[3,8]$ or necrosis [4], and rarely even visual field defects $[9,10]$ with eventual blindness [11], have been described by previous authors. This benign condition

*Corresponding author: Vaishnavi Batmanabane, Department of Retina and Vitreous, Chaithanya Eye Hospital and Research Institute, Kesavadasapuram, Trivandrum, Kerala 695004, India, Tel: +91 04712447183 ; Fax: +91 04712993985; E-mail: vaishb@gmail.com

Citation: Batmanabane V, Soman M, Nair U (2015) Management of Optic Disc Melanocytoma Associated with Choroidal Neovascularisation and Macular Detachment. J Ophthalmic Clin Res 2: 017.

Received: November 10, 2015; Accepted: December 10, 2015; Published: December 24, 2015 warrants close monitoring indefinitely due to the distinct, albeit small, possibility of the lesion turning malignant [3].

Ocular melanocytoma arises from the melanocytes of the Retinal Pigment Epithelium (RPE) and are usually located in the posterior pole. Often the full histopathological extent of the lesion might not be immediately obvious as it burrows beneath the retinal layers well past its exposed surface. This lesion has also been called a magnocellular nevus of the optic disc, stressing its benign nature.

Most melanocytomas are situated on the optic disc and if the patient is fortunate, will remain unobtrusive. Melanocytomas are rarely associated with secondary Choroidal Neovascular Membranes (CNVM) which may develop as a result of the neoplastic process breaching the integrity of the RPE. Once commenced, the neovascular process can threaten the fovea depending on its location. Subretinal haemorrhage and exudation will follow the occurrence of a CNVM, often causing a drastic worsening in vision. Different methods of managing CNVM in melanocytoma have been reported, ranging from photodynamic therapy to submacular surgery [12-14]. The discovery of anti-vascular Endothelial Growth Factor (VEGF) molecules has been a boon to the management of sight threatening intraocular neovascular processes. These agents target VEGF receptors, ultimately causing a decrease in angiogenesis. The current therapeutic benefit of anti-VEGF drugs like ranibizumab is that these agents can attack the inciting angiogenic factors without much collateral damage, yet as it is an invasive procedure, absolute certainty of the diagnosis and the benign nature of the lesion is necessary before the decision to treat with intravitreal injections is made.

We present the case of a 29 year old lady with an incidental melanocytoma of the optic disc and secondary CNVM, managed with intravitreal ranibizumab (Lucentis). The images that were obtained detailed the tracking of the melanotic pigments subretinally during and following treatment.

\section{Case Presentation}

A 28 year old lady presented to us with complaints of sudden defective vision in the left eye (OS) of four days duration. She had no previous ophthalmic examination elsewhere prior to presentation. Her Best Corrected Visual Acuity (BCVA) was $6 / 6$ in the right eye (OD) and 6/12 in OS. Anterior segment evaluation was within normal limits bilaterally. Fundus examination of OD was normal with a normal peripheral and posterior retina. OS showed a brownish black, horizontally oval lesion, measuring approximately $2 \mathrm{~mm} \times 2.5 \mathrm{~mm}$ in size situated on the optic disc (Figure 1a). The anterior surface of the lesion was exposed to the vitreous cavity while the temporal margins were blurred suggesting that the lesion extended beyond its visible margin. This exposed surface was uneven and excavated. The lesion seemed to be choking the disc, with the nasal disc margin appearing edematous, and the retinal veins appearing tortuous and engorged as compared to the other eye. A rim of Subretinal Fluid (SRF) was present surrounding the optic disc (Figure $1 \mathrm{~b}$ ) and involving the macula. A focus of subretinal fibrin was present one disc diameter nasal to the fovea with surrounding subretinal haemorrhage, suggestive of a neovascular process. There 
were flecks of black pigment dusted over the retina inferior to the disc suggesting that pigment had detached from the main lesion and settled over the retina. Pigment migration could also be discerned subretinally, extending in a linear fashion downward from the optic disc. There was no orange pigmentation over the lesion which could suggest a malignancy. A visual field analysis using the HFA 30-2 protocol was performed and a nasal scotoma extending from the blind spot and involving fixation was seen. Spectral Domain-optical Coherence Tomography (SD-OCT) of the lesion (HRA+OCT Spectralis, Heidelberg Engineering) showed intense hyper reflectivity of the lesion which was extending subretinally temporal to the optic nerve head and measuring $3.2 \mathrm{~mm}$ in horizontal and $2.9 \mathrm{~mm}$ in vertical dimensions (Figures 1c-e). The posterior extent of the lesion could not be recorded as the pigment was highly reflective, casting a shadow over the deeper structures. Line scan of the macula revealed SRF which was clear for the most part but had hyper reflectivity corresponding to the area of fibrin suggestive of exudation (Figure 1e). There was SRF surrounding the optic nerve head and extending approximately 2 disc diameters inferior to it. Fundus Fluorescein Angiography (FFA) documented a hypofluorescent mass which remained hypofluorescent through all the angiographic phases (Figures 2a-f). But an adjacent area showed a fuzzy hyperfluorescence with leak in the late frames of the angiogram suggestive of a secondary type 2 CNVM. Autofluorescence imaging of the lesion revealed complete blockage of reflectance suggestive of an infiltrative lesion on the optic disc eroding anteriorly which corresponded to the exposed part of the melanocytoma seen clinically. Surrounding this was an area of hypoautofluorescence which corresponded to the part of the lesion which had burrowed intraretinally on OCT. Ultrasound B scan revealed a homogenously solid hyperechoiec lesion on the optic nerve head approximately $1.8 \mathrm{~mm} \times 2 \mathrm{~mm}$. The lesion was too small to demonstrate high internal reflectivity as is classically described. There was no associated choroidal excavation. Based on these observations, a diagnosis of optic disc melanocytoma and secondary CNVM was made.

The patient was treated with $1.25 \mathrm{mg}$ of intravitreal injection ranibizumab (Lucentis) $0.05 \mathrm{ml}$ in view of the active CNVM. One week after the injection, the subretinal haemorrhage and fibrin had diminished with the resolution of most of the SRF, leaving a small residual amount around the optic disc (Figures 3a-d). Her vision had improved to $6 / 6$ in OS. Interestingly, we observed that there was a migration of pigments toward the fovea and inferiorly with the resolution of the fluid imaged with the SD-OCT and autofluorescence imaging (Figures $4 \mathrm{a}$ and $\mathrm{b}$ ). At last follow up 6 months later, her BCVA was $6 / 6$ in both eyes with fundus of OS showing a scarred CNVM with no subretinal fluid under the fovea, persistent disc edema and dispersed pigments from the exposed melanocytoma. The dimensions of the melanocytoma were constant with no change in size noted.

\section{Discussion}

This case report illustrates the advantage of intraocular imaging in diagnosis and management of complicated ocular melanocytoma. Melanocytomas are likely to be unilateral, with preponderance in the female gender [3], as in our patient. One large series reported that visual symptoms were unusual [3], but our patient had visual complaints due to the development of a CNVM, prompting her visit to the ophthalmologist. It is well documented that even small optic disc melanocytomas can cause visual dysfunction by mechanical effects: optic disc compression causing vascular occlusions or nerve fiber layer defects $[10,15]$. There are also secondary effects causing

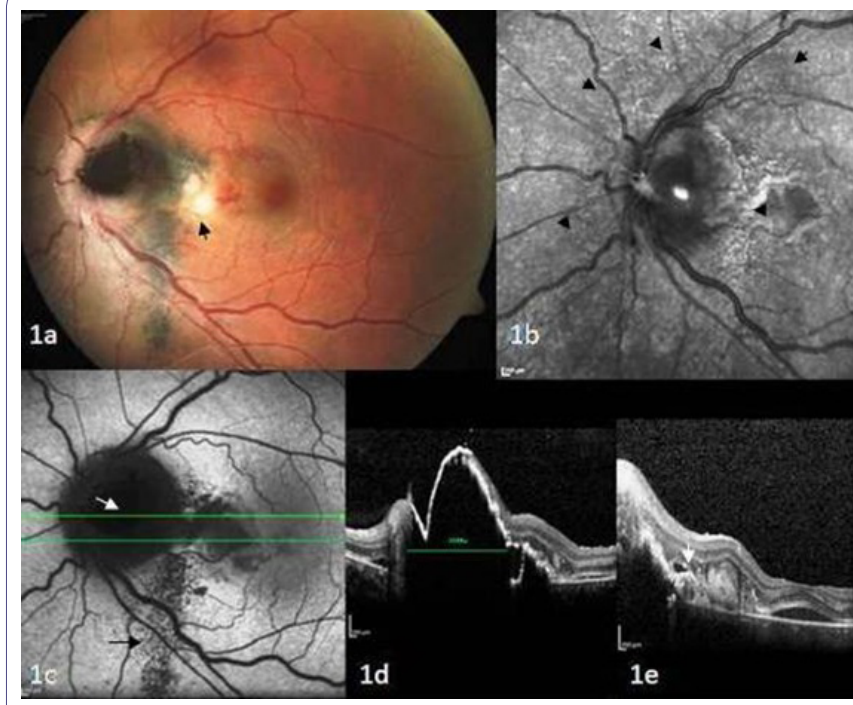

Figure 1: a) Clinical features of left fundus at presentation. Note the pigmented melanocytoma on the optic disc with surrounding edema. Subretinal fibrin (black arrow) with adjacent subretinal hemorrhage is obvious; b) Infra-red image of the melanocytoma. The subretinal fluid surrounding the optic disc can be visualized (black arrow heads); c) Autofluorescence image of the optic nerve head lesion. The retinal component of the melanocytoma is intensely hypoautofluorescent (white arrow). The subretinal pigments tracking inferiorly from the lesion can also be seen (black arrow); d) SD-OCT image of the cross section at line $d$ in 1c. The raised hyperreflective lesion on the optic nerve head with posterior back shadowing can be seen. Approximate horizontal diameter of the lesion is $2665 \mu \mathrm{m}$. The retinal and choroidal components can be clearly seen; and e) SD- OCT image of the cross section at line $e$ in 1c. Subretinal fluid with fibrin and hyper reflective point echoes (white arrow) lining the detachment suggestive of migrated pigment is well visualized.

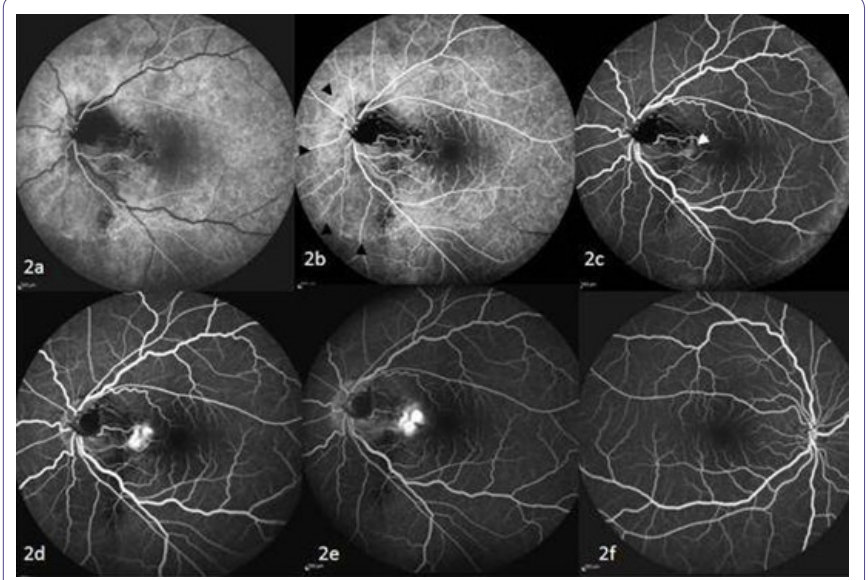

Figure 2: Fundus fluorescein angiogram of OS

a) Arterial phase: Note the blocked fluorescence of the melanocytoma at the optic disc and the migrated pigments inferiorly; b) Mid venous phase showing normal foveal avascular zone. The extent of the retinal peripapillary detachment is demarcated (black arrow heads); c) Fuzzy hyperfluorescence of the associated secondary CNVM (white arrow); d,e) Increasing hyperfluorescence of the CNVM with pooling in the subretinal space; and f) Normal right eye.

visual disturbances such as tumor necrosis causing ischemic optic neuropathy [16] or CNVM development. Clinically, differentiating these tumors from melanomas can pose a challenge in spite of available guidelines to help make a clinical diagnosis [17]. Our patient had a characteristic solitary pigmented lesion on the optic disc which is virtually the pathognomonic presentation of melanocytomas. Yet there were other features which raised red flags 


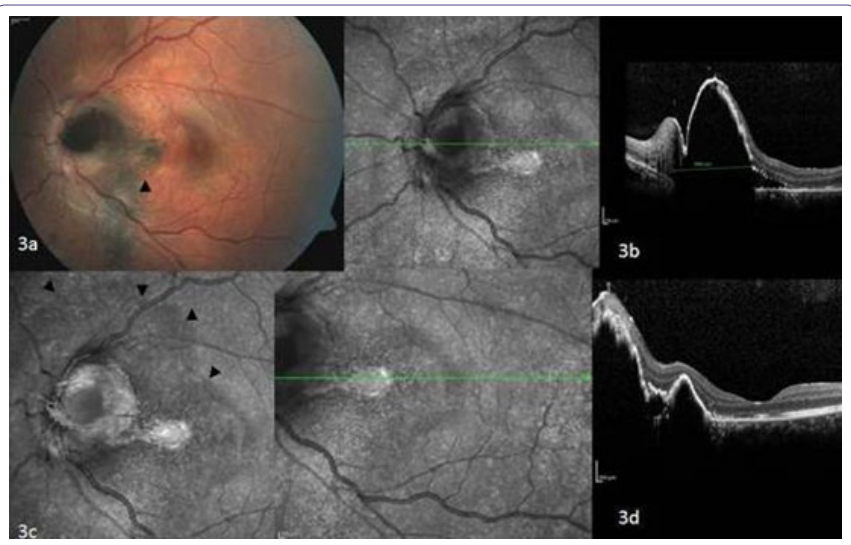

Figure 3: Features at follow-up after intravitreal ranibizumab.

a) Clinical features of left fundus 1 month later. The exposed melanocytoma shows no increase in size. There is resolution of the subretinal fibrin and the adjacent subretinal hemorrhage. The scarred CNVM can now be visualized (black arrowhead); b) Infra-red image and SD-OCT image of the cross section at the indicated line. The dimensions of the melanocytoma are unaltered but the surrounding subretinal fluid is reduced; c) Infrared imaging showing the eroded exposed anterior component of the melanocytoma. A line indicating RPE alteration in the region of the previous retinal detachment is seen (black arrowheads); and d) Infra-red image and SD-OCT image of the cross section at the indicated line. The macula is attached with mild RPE alteration.

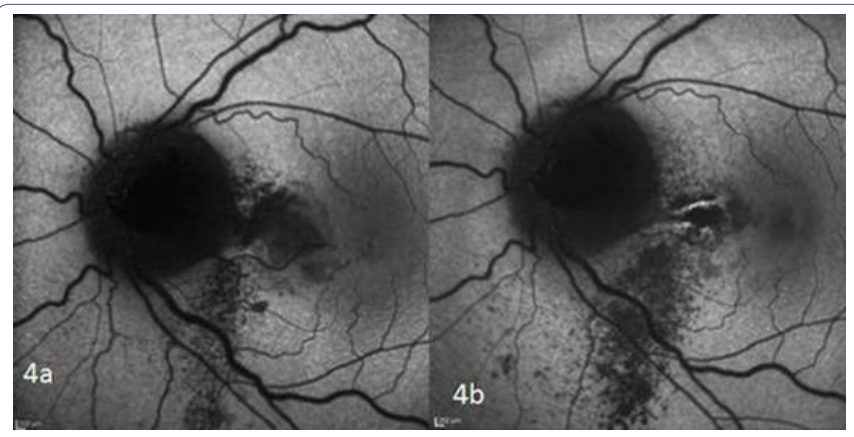

Figure 4: Pre and post treatment comparisons of autofluorescence. Note the obvious increase in the pigment tracking down from the optic nerve head lesion. The scarred CNVM can be visualized as a linear hyperfluorescence nasal to the fovea in $4 b$.

such as dimensions more than $2 \mathrm{~mm}$, subretinal fluid (which can be indicative of malignant change [18]) surrounding the lesion and associated hemorrhage. A papillary or juxtapapillary choroidal melanoma is the closest differential diagnosis but it is often a challenge to differentiate these neoplasms from choroidal nevi, or juxtapapillary adenomas or hyperplasia of the retinal pigment epithelium. We could confirm a diagnosis of melanocytoma in our patient by SD-OCT and B-scan ultrasonography. The presence of a perifoveal secondary CNVM was confirmed by the FFA. This rare cause of visual dysfunction is a late warning sign of poor visual prognosis if it involves the macula. These secondary CNVMs are easily imaged by FFA and warrant prompt intervention. Previous authors have described the use of Photodynamic Therapy (PDT) [12], intravitreal pharmacotherapy with bevacizumab [14] and submacular surgery [13] for CNVM removal. In all of these patients the visual outcome encouraging, as the CNVM was managed before it caused permanent macular photoreceptor damage. Non-invasive therapy such as PDT, is best chosen for patients in whom there is the slightest doubt about malignant transformation, as this is the safest treatment for these eyes. But the cost of the treatment might be limiting factor. In other cases where there is no uncertainty in diagnosis, intravitreal anti-VEGF therapy is convenient as it is effective. The only concern is if the needle tract will be susceptible to involvement if the melanocytoma does undergo a malignant transformation. One of the common effects of mechanical compression can be a central retinal vein occlusion. Clinically, she also seemed to have a 'choked disc', which led us to consider the possibility of mechanical compression at the optic cup. This can also occur due to perilesional inflammation or tumor necrosis [19]. In our case, although there was optic disc edema and dilatation of the central retinal veins, the FFA did not show any evidence of decreased or delayed retinal vein filling or vascular perfusion although the veins appeared more tortuous when compared to the opposite side.

The results after the first injection were encouraging with a 2 line visual improvement and clinical resolution of the subretinal fluid confirmed by OCT. Of concern was the migration of the pigment in the subretinal space, toward the fovea, which was a risk that had to be taken. This tracking was well imaged by autofluorescence imaging. As there has been subjective improvement in vision and clinical improvement with regard to the CNVM, we can assume that intravitreal ranibizumab had achieved the therapeutic goal in this patient.

The clinical dilemma of whether a pigmented optic disc lesion is malignant or not can be resolved by correlating clinical features and advanced imaging techniques like SD-OCT. This will determine the decision of how to treat the secondary neovascular processes. In conclusion, we report that appropriate imaging and intravitreal anti-VEGF agents may be considered to manage secondary CNVM in cases of optic disc melanocytoma, with good therapeutic outcomes.

\section{Acknowledgement}

We thank the Department of Retina and Vitreous for the use of the imaging equipment and support.

\section{References}

1. Eldaly H, Eldaly Z (2015) Melanocytoma of the optic nerve head, thirty-month follow-up. Semin Ophthalmol 21: 1-6.

2. Shukla SY, Shields JA, Eagle RC, Shields CL (2012) Transformation of optic disc melanocytoma into melanoma over 33 years. Arch Ophthalmol 130: 1344-1347.

3. Shields JA, Demirci H, Mashayekhi A, Shields CL (2004) Melanocytoma of optic disc in 115 cases: the 2004 Samuel Johnson memorial lecture, part 1. Ophthalmology 111: 1739-1746.

4. Lee E, Sanjay S (2015) Optic disc melanocytoma report of 5 patients from Singapore with a review of the literature. Asia Pac J Ophthalmol (Phila) 4: 273-278.

5. Agarwal S, Shanmugam MP, Gopal L, Krishnakumar S, Biswas J (2005) Necrotic melanocytoma of the optic disk with central retinal vascular obstruction. Retina 25: 364-367.

6. Thanos A, Gilbert AL, Gragoudas ES (2015) Severe vision loss with optic disc neovascularization after hemiretinal vascular obstruction associated with optic disc melanocytoma. JAMA Ophthalmol 133: 151502.

7. Besada E, Shechtman D, Barr RD (2002) Melanocytoma inducing compressive optic neuropathy: The ocular morbidity potential of an otherwise invariably benign lesion. Optometry 73: 33-38.

8. Shields JA, Demirci H, Mashayekhi A, Eagle RC Jr, Shields CL (2006) Melanocytoma of the optic disk: a review. Surv Ophthalmol 51: 93-104.

9. Punjabi OS, Lin CF, Chung HS, Gill MK (2011) Melanocytoma of the optic disc associated with visual field defects: clinical features and imaging characteristics. Ophthalmic Surg Lasers Imaging 42: 75-80. 
10. Osher RH, Shields JA, Layman PR (1979) Pupillary and visual field evaluation in patients with melanocytoma of the optic disc. Arch Ophthalmol 97 1096-1099.

11. Shields JA, Shields CL, Ehya H, Dhaliwal RS (2005) Total blindness from presumed optic nerve melanocytoma. Am J Ophthalmol 139: 1113-1114.

12. Chalam KV, Gupta SK, Shah GY, Agarwal S (2006) Successful management of melanocytoma-associated choroidal neovascularization with photodynamic therapy. Eur J Ophthalmol 16: 776-778.

13. Tran HV, Bovey EH, Uffer S, Zografos L (2006) Peripapillary choroidal neovascularization associated with melanocytoma of the optic disc: a clinicopathologic case report. Graefes Arch Clin Exp Ophthalmol 244: 1367-1369.

14. Urrets-Zavalia JA, Crim N, Esposito E, Correa L, Gonzalez-Castellanos ME et al. (2015) Bevacizumab for the treatment of a complicated posterior melanocytoma. Clin Ophthalmol 9: 455-459.
15. Shields JA, Shields CL, Lavrich J (2002) Melanocytoma of optic disk in a patient with neurofibromatosis 2. Retina 22: 222-223.

16. Al-Rashaed S, Abboud EB, Nowilaty SR (2010) Characteristics of optic disc melanocytomas presenting with visual dysfunction. Middle East Afr J Ophthalmol 17: 242-245.

17. Shields CL, Shields JA (2002) Clinical features of small choroidal melanoma. Curr Opin Ophthalmol 13: 135-141.

18. Mansour AM, Zimmerman L, La Piana FG, Beauchamp GR (1989) Clinicopathological findings in a growing optic nerve melanocytoma. $\mathrm{Br} \mathrm{J}$ Ophthalmol 73: 410-415.

19. Font RL, Chaqués-Alepuz V (2011) [Giant optic disk melanocytoma complicated with massive intraocular seeding]. Arch Soc Esp Oftalmol 86: 158-161. 